\title{
Uso de pantallas en niños pequeños en una ciudad de Argentina
} Screen use among young children in a city of Argentina

\author{
Dra. Ingrid Waisman ${ }^{a}$ Dra. Elisa Hidalgo y Dra. María L. Rossia
}

\section{RESUMEN}

La utilización de dispositivos móviles se ha generalizado en las familias, en todas las clases sociales y desde edades tempranas.

Objetivos: Evaluar el uso de pantallas fijas y móviles en niños pequeños, el tiempo dedicado a actividades infantiles tradicionales y su relación con el nivel educativo materno.

Material y métodos: Diseño descriptivo, transversal. Población: niños de 6 meses a 5 años atendidos en Río Cuarto, Córdoba, de julio a septiembre de 2016.

Variables: demográficas, ingreso familiar, edad de comienzo, frecuencia de uso, minutos diarios, otras actividades.

Resultados: Seincluyeron 160 encuestas; $99 \%$ de los hogares tenía televisión y 98,75\%, smartphones. Promedio de aparatos electrónicos: 5,68; los de menores ingresos:5,1 (DE 1,57); y los de mayores ingresos: 6,32 (DE 1,18) ( $\mathrm{p}=0,0000)$.

Antes de los 2 años, 80,3\% de los niños miraba televisión y 37,4\% utilizaba pantallas táctiles con ayuda. Entre 2 y 4 años, 38,7\% utilizaba pantallas sin ayuda.

El $93 \%$ de los niños miró televisión y el 56\% utilizó pantallas móviles con mucha frecuencia. Miraron un promedio de 75,6 minutos diarios de televisión y utilizaron otras pantallas 31,3 minutos; la lectura ocupó 20,4 minutos.

Las madres con estudios terciarios dedicaron más tiempo a la lectura de libros que las que tenían estudios secundarios o primarios (análisis de la varianza: $p=0,00007$ ).

Conclusiones: Elequipamiento tecnológico delos hogares es prácticamente universal; la exposición de los niños a las pantallas es temprana. La televisión es más utilizada, pero las pantallas móviles ocupan un tiempo importante. Las madres con estudios superiores dedican más tiempo a la lectura.

a. Neoclínica, Río Cuarto, provincia de Córdoba.

Correspondencia:

Dra. Ingrid Waisman: ingridwaisman@arnet. com.ar

Financiamiento: Ninguno que declarar.

Palabras clave: pantallas táctiles, televisión, tiempo de exposición, primera infancia.

http:/ / dx.doi.org/10.5546/aap.2018.e186 Texto completo en inglés:

http:/ / dx.doi.org/10.5546/ aap.2018.eng.e186

Cómo citar: Waisman I, Hidalgo E, Rossi ML. Uso de pantallas en niños pequeños en una ciudad de Argentina. Arch Argent Pediatr 2018;116(2):e186-e195.

\author{
"Tanta soledad, todos conectados, \\ niños de pantalla, \\ bienvenidos al mercado". \\ (Canción popular, \\ letra de Andrés Ciro Martínez)
}

\section{INTRODUCCIÓN}

El impacto de la tecnología en la vida del hombre está en aumento permanente y los niños no son ajenos a esta realidad. Desde hace más de 40 años, los medios más tradicionales de comunicación, como la televisión y la radio, ocupan un lugar importante en las actividades cotidianas de las familias, pero en los últimos años, la aparición de tablets y smartphones ha cambiado la forma de comunicarse, informarse y entretenerse. La utilización de dispositivos móviles y pantallas táctiles se ha generalizado, habitualmente, con independencia del estatus económico o social de las familias. La cultura de las pantallas ha penetrado y permea todas las clases sociales y se utiliza a edades cada vez más tempranas. ${ }^{1,2}$

Los dispositivos móviles presentan indudables atractivos: su portabilidad, la comodidad para los padres en cuanto a entretener a los niños mientras ellos realizan tareas necesarias, el acceso a innumerables fuentes de entretenimiento e información, y la disminución relativa de costos. ${ }^{3}$ Los niños, para bien o para mal, tienen acceso a todo tipo de pantallas a edades cada vez más tempranas, $y$, aunque pareciera que esta tendencia es inexorable, aún no están claras las ventajas y las desventajas de este contacto anticipado.

Los programas de televisión o de medios interactivos bien diseñados 
pueden servir de juego y entretenimiento a los niños menores, aunque, para aprender de las pantallas, se requiere interacción y acompañamiento de los adultos. ${ }^{4}$

La exposición a las pantallas en los niños tiene desventajas. Entre las principales, están la asociación con sedentarismo y obesidad, la disminución de las horas de sueño, la posible dificultad en la interacción con otras personas, la afectación negativa de algunos rasgos de la conducta y la exposición a contenidos inapropiados. ${ }^{5}$

También se debe considerar el menor tiempo dedicado a otras actividades, como la lectura de libros por parte de niños y padres, menor cantidad de actividades al aire libre y menor cantidad de contactos interpersonales.

Es interesante focalizarse en la niñez temprana, antes de que los niños utilicen las redes sociales, pues es cuando se establecen hábitos relacionados con el uso de los medios para la vida futura; también es a esta edad en que los padres juegan un rol importante en los hábitos de los niños en relación con los medios. Este tiempo es un período de enorme plasticidad cerebral, cuando las experiencias ejercen profundas influencias sobre el desarrollo social, cognitivo y emocional, y en el cual son establecidas rutinas relacionadas con la salud, como comer, dormir y realizar actividad física. ${ }^{4}$

Existen varios estudios epidemiológicos y normativas respecto al uso de pantallas en niños pequeños en países del primer mundo, ${ }^{3-8}$ pero no se ha encontrado un enfoque similar para ese grupo etario en Latinoamérica o en Argentina.

Los objetivos de este trabajo son evaluar el uso de pantallas fijas y móviles en niños pequeños en una ciudad de Argentina, el tiempo dedicado a otras actividades infantiles tradicionales y su relación con el nivel educativo de las madres.

\section{MATERIAL Y MÉTODOS}

Diseño. Trabajo observacional, descriptivo, prospectivo, transversal.

Población. Pacientes de 6 meses a 4 años, 11 meses y 29 días de edad, y sus madres, atendidos en los Consultorios Externos de Neoclínica, Río Cuarto, Córdoba, entre los meses de julio a septiembre de 2016.

La institución donde se realizó el estudio es una clínica para la atención de pacientes de obras sociales y privados, con especial enfoque en el área materno-infantil. Posee servicios de internación de Pediatría, Neonatología,
Ginecoobstetricia y de otras especialidades. El área de Consultorios Externos de Pediatría se encuentra en un espacio edilicio propio y cuenta con Consultorios de Pediatría general, de especialidades pediátricas y un Consultorio de Atención Permanente.

Cálculo muestral. Para una prevalencia estimada de uso de pantallas táctiles del 50\%, el estadístico arroja una muestra de 159 encuestas.

Criterios de inclusión: pacientes de 6 meses a 4 años, 11 meses y 29 días atendidos en los Consultorios Externos de Pediatría o en el Consultorio de Atención Permanente.

Criterios de exclusión: niños con problemas neurológicos o discapacidad de cualquier tipo.

\section{Métodos}

Se confeccionó una encuesta con preguntas cerradas, adaptada de la encuesta realizada por Common Sense Media. ${ }^{6}$ Se invitó a las madres a participar y se confirmó su consentimiento con su firma en el formulario (Anexo 1).

Durante el mes de mayo de 2016, se aplicó una encuesta piloto a 15 madres, y se corrigieron algunas preguntas que resultaron poco claras.

\section{Variables}

Edades de niños, edades de madres y años de estudio de las madres. Se consideró secundario incompleto menos de 12 años de estudio; secundario completo: de 12 a 16 años de estudio; universitario completo: 17 años de estudio o más.

Ingreso familiar: menor de 20000 pesos mensuales, 20000 pesos mensuales o mayor.

Aparatos electrónicos en el hogar: televisión, computadora, tablet, smartphone, videojuegos (ninguno, uno, dos o más).

Desde qué edad usaba pantallas con ayuda y sin ayuda: menor de un año, de 1 a 2 , de 2 a 3 , de 3 a 4 , más de 4 .

Frecuencia semanal de uso: nunca, una por semana, varias por semana, diaria. La frecuencia se aplicó a cada una de las pantallas y al conjunto, y también a otras actividades: lecturas, aire libre, otro tipo de juegos. Se dicotomizó esta variable en uso poco frecuente (nunca o una vez por semana) y uso frecuente (varias por semana, diariamente).

Puntajes de uso semanal. Se consideró lo siguiente para este puntaje: 1) la utilización de televisión, tablet y smartphone; 2) aire libre, lecturas y otros juegos de representación.

Nunca (0 puntos), 1 vez por semana (2 puntos), varias veces (4 puntos), diariamente (6 puntos), para establecer medias y medianas. 
Minutos diarios: televisión, pantallas táctiles (tablet y smartphone), lectura de cuentos.

Análisis: se confeccionó la base de datos en Excel y se analizó con Epi Info 7.1.4.0, marca de los Centers for Disease Control and Prevention (CDC).

Las variables numéricas se analizaron comparando sus promedios y proporciones, mediante la aplicación de la prueba z y el análisis de la varianza (analysis of variance; ANOVA, por sus siglas en inglés); se definió significativo como $<0,05$.

Se calcularon odds ratio para demostrar la asociación entre variables cualitativas, teniendo en cuenta un índice de confianza del $95 \%$.

\section{RESULTADOS}

Se incluyeron en forma consecutiva las encuestas respondidas por las madres. No hubo rechazos a participar. Se completaron 160 encuestas. La proporción de género en los niños fue $1: 1$.

\section{Datos generales}

En la Tabla 1, se observan los datos demográficos de madres y niños.
El ingreso familiar fue menor de $\$ 20000$ en 93 encuestadas $(58,1 \%)$ y $\$ 20000$ o mayor en 67 $(41,8 \%)$.

La mayoría de los hogares (99\%) contaba con televisión, y, de ellos, $76,8 \%$, con 2 o más aparatos. En 75,6\%, había una o dos computadoras, y 98,75\% poseían teléfonos celulares inteligentes, y la gran mayoría de las familias, dos o más aparatos.

El promedio de aparatos electrónicos en el hogar fue de 5,68. Para los hogares de menores ingresos, la media fue de 5,1 (desvío estándar -DE-1,57) y, para los de mayores ingresos, de 6,32 (DE 1,18). La diferencia fue significativa $(p=0,0000)$.

\section{Frecuencia y tiempo de uso. Comparación con otras actividades}

El porcentaje de tiempo que los niños dedicaron semanalmente a cada una de las siguientes tres actividades (televisión, tablet/ smartphone y lectura) puede observarse en la Tabla 2.

Para confeccionar la Tabla 3, se sumaron los puntajes y se calcularon medias y medianas del uso semanal de pantallas (televisión, tablet y smartphone) y se los comparó con los puntajes de

Tabla 1. Datos demográficos $(\mathrm{N}=160)$

\begin{tabular}{|c|c|c|c|c|c|}
\hline & & & & Frecuencia & Porcentaje \\
\hline \multirow[t]{7}{*}{ Niños } & Grupos de edad & $\mathrm{A}$ & 6-11 m. & 16 & 10 \\
\hline & & B & $12-23 \mathrm{~m}$. & 38 & 23,7 \\
\hline & & $\mathrm{C}$ & $24-35 \mathrm{~m}$. & 39 & 24,4 \\
\hline & & $\mathrm{D}$ & $36-47 \mathrm{~m}$ & 36 & 22,5 \\
\hline & & $\mathrm{E}$ & $48-59 \mathrm{~m}$. & 31 & 19,4 \\
\hline & Edad en meses & Media & DE & Mediana & Rango \\
\hline & & 31,3 & 14,29 & 30,5 & $6-59$ \\
\hline \multirow[t]{2}{*}{ Madres } & Edad en años & 30,16 & 5,54 & 31 & $18-45$ \\
\hline & Años de estudio & 13,43 & 4,06 & 13 & $6-22$ \\
\hline
\end{tabular}

DE: desvío estándar; m.: meses.

TABLA 2. Porcentaje de tiempo semanal dedicado a diferentes actividades

\begin{tabular}{lccccc}
\hline & \multicolumn{4}{c}{ Frecuencia } \\
\cline { 2 - 5 } & Diaria & Varias por semana & Una por semana & Nunca & Total de porcentaje \\
\hline Televisión & 77 & 17 & 3 & 32 & 100 \\
Pantallas & 34 & 22 & 12 & 100 & 21 \\
Libros & 25 & 38 & 16 & 100 \\
\hline
\end{tabular}

TABla 3. Puntaje de uso semanal de pantallas versus otras actividades

\begin{tabular}{|c|c|c|c|c|c|c|c|c|c|}
\hline & Observaciones & Total & Media & $\mathrm{DE}$ & Min. & $25 \%$ & Mediana & $75 \%$ & Máx. \\
\hline Televisión, tablet, smartphone & 160 & 1644 & 10,275 & 3,79 & 0 & 8 & 10 & 12 & 18 \\
\hline Aire libre, juegos y libros & 160 & 1936 & 12,1 & 4,0859 & 0 & 10 & 12 & 16 & 18 \\
\hline
\end{tabular}

DE: desvío estándar. 
todas las otras actividades recreativas (lectura, actividades al aire libre y otros juegos).

La correlación entre el uso de pantallas y otras actividades se muestra en la Tabla 4.

Las comparaciones entre el tiempo semanal dedicado a ver televisión, el tiempo dedicado a la lectura y al uso de otras pantallas se observan en la Tabla 5.

En relación con la exposición a las pantallas, el 93\% de los niños miró televisión con mucha frecuencia, y el 56\% utilizó pantallas móviles con mucha frecuencia. Se definió "mucha frecuencia" el uso diario o varias veces por semana.

Al medirlo en minutos, los niños miraron un promedio de 75,6 minutos diarios de televisión y utilizaron otras pantallas 31,3 minutos, mientras que la lectura ocupó 20,4 minutos.

\section{Uso de pantallas en relación con la edad}

Antes de los 2 años, el 80,3\% de los niños miraba televisión con ayuda de los padres, y el $37,4 \%$ utilizaba con ayuda pantallas táctiles (tablet o smartphone).

Entre los 2 y los 4 años, 28,7\% podían mirar televisión sin ayuda, y 38,7\% podían utilizar sin ayuda pantallas táctiles.

Se analizó la distribución del tiempo semanal según las edades de los niños que componían la muestra. La frecuencia semanal por edades de uso de televisión, pantallas móviles y libros se observa en la Figura 1.

Los minutos utilizados el día anterior para ver televisión, otras pantallas y libros según grupos de edad menores de 2 años o 2 años y mayores se observan en la Tabla 6.

\section{Lectura y nivel educativo materno}

El puntaje semanal de lectura de libros tuvo una media y DE de 2,16 $\pm 2,1$ cuando el nivel de la madre fue educación primaria o secundaria incompleta; 3,51 $\pm 2,1$ para secundario completo y $4,12 \pm 1,5$ para universitario completo. El análisis de ANOVA mostró un valor de $p=0,00007$.

\section{DISCUSIÓN}

Los datos demográficos mostraron un grupo socioeconómico de clase media, un nivel educativo en las madres de medio a medio alto y la presencia de televisión y pantallas en prácticamente todos los hogares. Pese a que la diferencia entre hogares de mayores y menores ingresos respecto a la tenencia de electrónicos fue significativa, prácticamente, todos poseían televisión y smartphones.

La exposición a la televisión fue siempre mayor que otras actividades, seguida por las pantallas móviles. Al sumar los puntajes de todas las actividades recreativas no relacionadas con pantallas, estos estuvieron ligeramente por encima del uso de pantallas, pero se debe tener en cuenta que era un grupo etario de niños muy pequeños, aún sin escolarizar, en los que juegos, actividades al aire libre y lectura/ escucha de cuentos ocupaban buena parte de su rutina diaria.

El mayor tiempo dedicado a la visualización de pantallas no se correlacionó con el tiempo dedicado a otras actividades, por lo que se considera que no existe relación directa entre esas variables.

En consonancia con otros autores,,$^{3,7,8}$ se encontró que la exposición a las pantallas era casi

TABLA 4. Coeficiente de correlación

\begin{tabular}{lcc}
\hline Variable $\mathbf{1}$ & Variable 2 & Coeficiente de correlación \\
\hline Todas las pantallas $\left(^{*}\right)$ & Todas las otras actividades $\left({ }^{* *}\right)$ & 0,00016246 \\
Televisión & Lectura & 0,00274 \\
Televisión & Actividades al aire libre & 0,0373 \\
\hline
\end{tabular}

$\left.{ }^{*}\right)$ Televisión, tablets y smartphones.

${ }^{* *}$ Aire libre, lecturas y otros juegos de representación.

TABla 5. Comparación de la frecuencia semanal de uso de televisión con la lectura y otras pantallas

\begin{tabular}{lcccc}
\hline & Muy frecuente & Poco frecuente & OR & $P$ \\
\hline Televisión & 150 & 10 & $8,76(4,2-17,9)$ & 0,0000 \\
Libros & 101 & 59 & $11,66(5,7-23,7)$ & 0,0000 \\
Otras pantallas & 90 & 70 & \\
\hline
\end{tabular}

OR: odds ratio. 
universal, y la adquisición de habilidades para su uso, muy temprana.

En un trabajo realizado por la Subcomisión de Tecnologías de Información y Comunicación de la Sociedad Argentina de Pediatría (SAP) entre 2007 y 2008, se analizaron niños mayores de 4 años y adolescentes. Se encontró que la mitad de ellos se conectaban diariamente $y$, en menos de la mitad de los casos, recibían supervisión de un adulto responsable acerca de los contenidos o de los contactos a los que estaban expuestos. La recomendación en esa oportunidad fue que los padres debían involucrarse en el cuidado de sus hijos en la Web, fomentar el diálogo fluido y estimular la generación de reglas consensuadas actuando con el ejemplo. ${ }^{9} \mathrm{La}$ introducción posterior en el mercado de tablets y de smartphones actualiza y reafirma esta recomendación y la hace extensiva a niños más pequeños.

La recomendación de la Asociación Americana de Pediatría (AAP) del año 2011, que, en general, fue adoptada por los pediatras y que aconsejaba no utilizar pantallas móviles antes de los 2 años, no parece realista. ${ }^{10}$ En noviembre de 2016, la misma Asociación recomendó para esas edades, un uso prudente en la duración de la exposición y, fundamentalmente, la supervisión y el monitoreo por parte de padres o cuidadores. ${ }^{11}$
Al considerar la frecuencia semanal, en nuestro trabajo, el uso de la televisión permaneció constante en todos los grupos de edad, mientras que el uso de pantallas móviles se incrementó con la edad. Sin embargo, al analizar los minutos diarios de exposición a la televisión, se constató que, a mayor edad, mayor era la exposición a esta pantalla. Este dato contradictorio podría explicarse por la menor capacidad de los niños más pequeños de fijar su atención: si bien miran televisión con frecuencia, la duración de esta actividad es menor.

Los niños muy pequeños se encuentran en una etapa de desarrollo sensoriomotor, y su comprensión del contenido bidimensional de las pantallas es limitado. Se cree que, en niños menores de 2 años, el control atencional y el pensamiento simbólico son inmaduros para que puedan transferir conocimientos desde una pantalla a la vida real en tres dimensiones. ${ }^{4,11}$

Antes de los dos años, los niños desarrollan habilidades cognitivas, sensoriales y adquisición de lenguaje; esto requiere que exploren el mundo que los rodea y una interacción adecuada con padres o cuidadores para adquirir una madurez exitosa. ${ }^{5}$ No está claro de qué modo afecta el uso generalizado de pantallas táctiles a estos procesos madurativos, pero hay acuerdo en distintos

Figura 1. Frecuencia semanal de uso según la edad (puntaje)

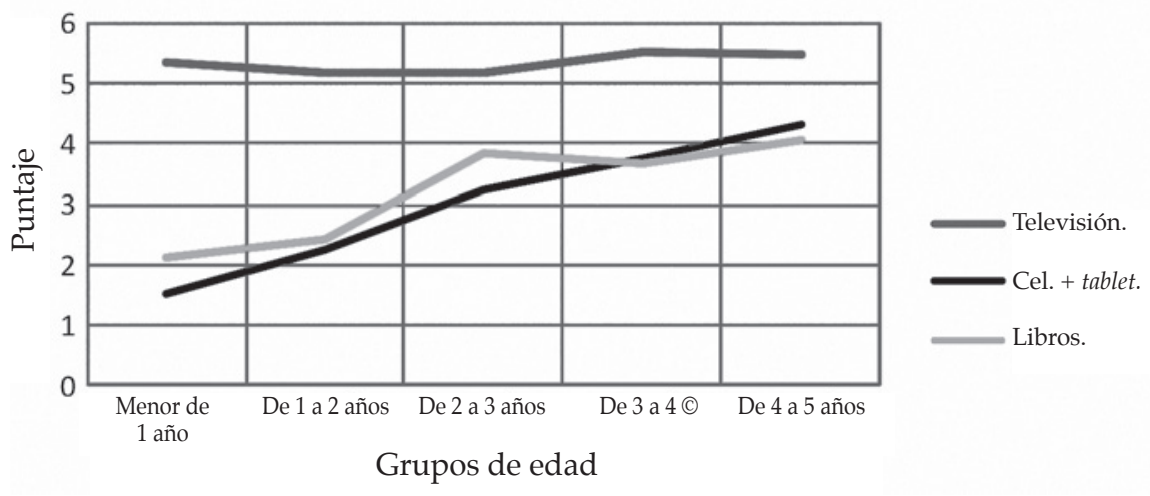

TABLA 6. Minutos utilizados para ver televisión, otras pantallas y libros según grupos de edad en un día

\begin{tabular}{lcccc}
\hline & \multicolumn{2}{c}{ Menor de 2 años } & \multicolumn{2}{c}{ 2 años o mayor } \\
\cline { 2 - 5 } & Media (DE) & Mediana (rango) & Media (DE) & Mediana (rango) \\
\hline Televisión & $46,66(45,5)$ & $30(0-180)$ & $90,28(60,6)$ & $60(0-180)$ \\
Otras pantallas & $16,34(34,4)$ & $0(0-150)$ & $39,1(47)$ & $20(0-150)$ \\
Libros & $13,14(31)$ & $0(0-150)$ & $24,05(35)$ & $20(0-150)$ \\
\hline
\end{tabular}

DE: desvío estándar. 
autores sobre la necesidad de que un adulto acompañe en el proceso, seleccione los contenidos y limite los tiempos de exposición. ${ }^{3,4,11,12}$

Según la AAP, la industria ha buscado al grupo de 0 a 2 años (y sus padres) como consumidores clave de los medios electrónicos. Se han diseñado programas televisivos, videos y música para este grupo etario. En la actualidad, la mayoría de los padres informan que sus hijos menores de 2 años utilizan algún tipo de medios electrónicos. ${ }^{7,11}$

Las pantallas fijas y móviles no son buenas ni malas. En el grupo etario que nos ocupa, la diferencia la hace la utilización que los padres hacen de ellas, cómo introducen a sus hijos en la cultura tecnológica y cómo los preservan de posibles riesgos. ${ }^{1}$

Cuando, a su vez, los padres utilizan en exceso los dispositivos móviles, esto se asocia con menor interacción verbal y no verbal en la familia, y puede llevar a conflictos entre padres y niños. ${ }^{4,5,13}$

Por ese motivo, desde la Subcomisión de Tecnologías de Información y Comunicación (TIC) de la SAP, se ha propuesto recientemente a la comunidad pediátrica promover la "cena sin pantallas" ${ }^{2}$ para incrementar, en un horario sensible para las familias, el contacto personal directo.

Otros autores ${ }^{3}$ hacen referencia a la posesión por parte del niño de distintos medios electrónicos. En nuestro medio, más bien se ha encontrado un uso familiar, tanto de la televisión como de las tablets y, muy especialmente, de los smartphones. Si bien no son propiedad de los niños pequeños, su disponibilidad es amplia.

La lectura de libros por parte de los padres o la escucha de cuentos, indudablemente, quedan relegados por las novedades tecnológicas. Sin embargo, se encontró que las madres con educación superior utilizaban más tiempo para contar cuentos o leer libros a sus hijos pequeños.

Las limitaciones de este trabajo son, por una parte, la pertenencia de los pacientes a un grupo social bastante homogéneo de clase media y con escolaridad materna elevada, lo que hace difícil extrapolar resultados a la población en general. ${ }^{3}$ Por otra parte, en varias de las respuestas, puede haber sesgo de recuerdo, especialmente, en aquellas que se refieren a la historia de utilización de los medios según la edad de los niños. Además, en consonancia con la preocupación de otros autores, ${ }^{14}$ las respuestas se basaron en la percepción materna y no en mediciones objetivas.
Este trabajo no pretende dilucidar el efecto de la televisión o de las pantallas móviles sobre el desarrollo de los niños menores de 5 años; solamente, describir una situación en nuestro país, que, con algunas características propias, se asemeja bastante a la realidad global, y llamar la atención de los pediatras, pues a esta edad, se establecen pautas de conducta que durarán por muchos años, no solo en relación con la utilización de medios digitales, sino en muchos aspectos de la vida y la salud del niño.

Los pediatras pueden constituirse en una adecuada fuente de información para los padres, comentando los resultados de las investigaciones realizadas, ofreciendo sugerencias para acompañar a los niños en el uso de las pantallas y aconsejando cómo regular el tiempo de exposición.

\section{Agradecimiento}

A la Dra. Paula Otero por la lectura preliminar del manuscrito y las valiosas sugerencias.

\section{REFERENCIAS}

1. Gutnick AL, Robb M, Takeuchi L, et al. Always connected: The new digital media habits of young children. New York: The Joan Ganz Cooney Center at Sesame Workshop; 2010. [Acceso: 6 de septiembre de 2017]. Disponible en: http: / / www.joanganzcooneycenter.org/wp-content/ uploads/2011/03/jgcc_alwaysconnected.pdf.

2. Campaña \#Cena sin Pantallas. Asociación Chicos.net. Tecnología para un Mundo Mejor. 2. ${ }^{\text {da }}$ ed. [Acceso: 6 de septiembre de 2017]. Disponible en http: / / www.chicos. net/2016/ cenasinpantallas/.

3. Kabali HK, Irigoyen M, Nunez-Davis R, et al. Exposure and Use of Mobile Media Devices by Young Children. Pediatrics. 2015;136(6):1044-50.

4. Radesky JS, Christakis DA. Increased Screen Time: Implications for Early Childhood Development and Behavior. Pediatr Clin North Am 2016;63(5):827-39.

5. Reid Chassiakos Y, Radesky J, Christakis D, et al. Children and Adolescents and Digital Media. Pediatrics 2016:138(5):e20162593.

6. Rideout V, Saphir M, Pai S, et al. Zero to Eight: children's media use in America 2013. Common Sense Media Research Study. [Acceso: 6 de septiembre de 2017]. Disponible en: https: / / www.commonsensemedia.org/research/zero-toeight-childrens-media-use-inamerica-2013.

7. Chandra M, Jalaludin B, Woolfender S, et al. Screen time of infants in Sydney, Australia: a birth cohort study. BMJ Open.2016;6(10):e012342.

8. Ahearne $\mathrm{C}$, Dilworth $\mathrm{S}$, Rollings $\mathrm{R}$, et al. Touch-screen technology usagein toddlers. Arch Dis Child2016;101(2):181-3.

9. Grupo de informática dela Sociedad Argentina de Pediatría. Resultado de la encuesta "Internet en los hogares". [Acceso: 6 de septiembre de 2017]. Disponible en: http:/ / www. sap.org.ar/docs / organizacion/Grupos / informatica / encuesta.pdf.

10. Council on Communications and Media, Brown A. Media Use by Children Younger Than 2 Years. Pediatrics 2011;128(5):1040-5.

11. Kostyrka-Allchorne K, Cooper N R, Simpson A. 
e192 / Arch Argent Pediatr 2018;116(2):e186-e195 / Artículo original

Touchscreen generation: children's current media use, parental supervision methods and attitudes towards contemporary media. Acta Paediatr 2017;106(4):654-62.

12. Linebarger DL, Barr R, Lapierre MA, et al. Associations between parenting, media use, cumulative risk, and children's executive functioning. I Dev Behav Pediatr 2014;35(6):367-77.

13. Richard G. Los niños frente a las pantallas. Boletín Especial
Día del Niño. Universidad Católica de Córdoba, 2016. [Acceso: 6 de septiembre de 2017]. Disponible en: http:/ / www2.ucc.edu.ar/ especiales/los-ninos-frente-a-laspantallas/.

14. Goh SN, Teh LH, Tay WR, et al. Sociodemographic, home environment and parental influences on total and devicespecific screen viewing in children aged 2 years and below: an observational study. BMJ Open. 2016;6(1):e009113. 


\section{ANEXO 1}

\section{Encuesta}

Encuesta N. ${ }^{\circ}$

Nombre del niño e inicial del apellido:

1) Datos demográficos

\begin{tabular}{|l|l|l|}
\hline & Años cumplidos & $\begin{array}{l}\text { Meses } \\
\text { cumplidos }\end{array}$ \\
\hline Edad & & \\
\hline Sexo & F & M \\
\hline Hijo n. $^{\circ}$ & & Único \\
\hline
\end{tabular}

\begin{tabular}{|l|l|l|l|}
\hline & Madre & Padre & \\
\hline Edad & & & \\
\hline Años de estudio & & & \\
\hline ¿Trabaja afuera? & & & \\
\hline Cantidad de hijos & & & \\
\hline $\begin{array}{l}\text { ¿Tiene acceso a } \\
\text { internet en casa? }\end{array}$ & Sí & NO & \\
\hline Ingreso familiar & $<20000$ & $20000-40000$ & $>40000$ \\
\hline
\end{tabular}

2) Pantallas en el hogar

¿Cuál de los siguientes aparatos (si hay alguno) existen en su hogar?

\begin{tabular}{|l|l|l|l|}
\hline & Ninguno & 1 & 2 o más \\
\hline \multicolumn{1}{|c|}{ Televisión } & & & \\
\hline Teléfono celular & & & \\
\hline Computadora & & & \\
\hline $\begin{array}{l}\text { Dispositivos móviles } \\
\text { (tablet, iPod) }\end{array}$ & & & \\
\hline Videojuegos & & & \\
\hline Otro & & & \\
\hline
\end{tabular}

3) ¿Cuál de los siguientes equipos está instalado en la habitación del niño?

\begin{tabular}{|l|l|l|}
\hline Equipo & Sí & NO \\
\hline Televisor & & \\
\hline Computadora o netbook & & \\
\hline Videojuegos & & \\
\hline Otro & & \\
\hline & & \\
\hline
\end{tabular}

4) ¿Cuál de los siguientes, si tiene alguno, posee el niño para sí mismo? Aunque no sea de su propiedad, dispone de él cuando quiere.

\begin{tabular}{|l|l|l|}
\hline Equipo & sí & NO \\
\hline Televisor & & \\
\hline
\end{tabular}


e194 / Arch Argent Pediatr 2018;116(2):e186-e195 / Artículo original

\begin{tabular}{|l|l|l|}
\hline Computadora o netbook & & \\
\hline Videojuegos & & \\
\hline Tablet & & \\
\hline Teléfono celular & & \\
\hline Otro & & \\
\hline
\end{tabular}

5) ¿A qué edad comenzó el niño?

\begin{tabular}{|l|l|l|l|l|l|l|l|l|l|l}
\hline \multirow{2}{*}{ Actividad } & \multicolumn{2}{l}{ Con ayuda } & \multicolumn{2}{l|l}{ Solo } \\
\cline { 2 - 11 } & $\begin{array}{l}<1 \\
\text { a. }\end{array}$ & 1 & 2 & 3 & 4 & $\begin{array}{l}<1 \\
\text { a. }\end{array}$ & 1 & 2 & 3 & 4 \\
\hline $\begin{array}{l}\text { Ver un programa en } \\
\text { televisión }\end{array}$ & & & & & & & & & & \\
\hline $\begin{array}{l}\text { Trabajar con } \\
\text { pantallas táctiles }\end{array}$ & & & & & & & & & & \\
\hline $\begin{array}{l}\text { Llamar a alguien por } \\
\text { tel. o pantalla }\end{array}$ & & & & & & & & & & \\
\hline Usar un videojuego & & & & & & & & & & \\
\hline Navegar por internet & & & & & & & & & & \\
\hline Usar aplicaciones & & & & & & & & & & \\
\hline
\end{tabular}

a.: año.

6) ¿Cuántas y cuáles apps utiliza el niño (con o sin ayuda)?

Cantidad:

Nombres que recuerde:

7) ¿Con qué frecuencia realiza las siguientes actividades?

\begin{tabular}{|l|l|l|l|l|}
\hline Actividad & Nunca & $\begin{array}{l}1 \text { vez por } \\
\text { semana }\end{array}$ & $\begin{array}{l}\text { Varias } \\
\text { veces por } \\
\text { semana }\end{array}$ & $\begin{array}{l}\text { Diariamen } \\
\text { te }\end{array}$ \\
\hline Ver televisión & & & & \\
\hline $\begin{array}{l}\text { Usar } \\
\text { computadora }\end{array}$ & & & & \\
\hline Usar videojuegos & & & & \\
\hline $\begin{array}{l}\text { Aplicaciones en } \\
\text { el teléf. o tablet }\end{array}$ & & & & \\
\hline $\begin{array}{l}\text { Leer libros o que } \\
\text { le lean }\end{array}$ & & & & \\
\hline $\begin{array}{l}\text { Activ. deportiva o } \\
\text { al aire libre }\end{array}$ & & & & \\
\hline $\begin{array}{l}\text { Juegos didácticos } \\
\text { o dibujar }\end{array}$ & & & & \\
\hline $\begin{array}{l}\text { Usar aplicaciones } \\
\text { educativas }\end{array}$ & & & & \\
\hline & & & & \\
\hline
\end{tabular}


8) ¿Durante cuánto tiempo realizó el niño AYER las siguientes actividades?

\begin{tabular}{|l|l|l|l|l|l|}
\hline Actividad & $\left\langle 30^{\prime}\right.$ & Media hora & 1 hora & 2 horas & Más de 2 h \\
\hline Ver televisión & & & & & \\
\hline $\begin{array}{l}\text { Usar } \\
\text { computadora }\end{array}$ & & & & & \\
\hline $\begin{array}{l}\text { Usar } \\
\text { videojuegos }\end{array}$ & & & & & \\
\hline $\begin{array}{l}\text { Aplicaciones } \\
\text { en el teléf. o } \\
\text { tablet }\end{array}$ & & & & & \\
\hline $\begin{array}{l}\text { Leer libros o } \\
\text { que le lean }\end{array}$ & & & & & \\
\hline $\begin{array}{l}\text { Activ. } \\
\text { deportiva o } \\
\text { al aire libre }\end{array}$ & & & & & \\
\hline $\begin{array}{l}\text { Juegos } \\
\text { didácticos o } \\
\text { dibujar }\end{array}$ & & & & & \\
\hline $\begin{array}{l}\text { Usar } \\
\text { aplicaciones } \\
\text { educativas }\end{array}$ & & & & & \\
\hline
\end{tabular}

9) Cuando el niño realiza otras actividades, ¿con qué frecuencia se encuentra la televisión encendida de fondo?

\begin{tabular}{|l|l|l|l|l|}
\hline $\begin{array}{l}\text { Televisión } \\
\text { encendida }\end{array}$ & \multicolumn{4}{|c|}{ Frecuencia } \\
\hline & Nunca & Rara vez & Frecuentemente & Siempre \\
\hline
\end{tabular}

10) ¿Con qué objeto su hijo utiliza estos dispositivos?

- Solo para entretenimiento.

- Para que los padres o cuidadores puedan cumplir actividades domésticas o laborales.

- Para entretenerlo en lugares públicos o viajes.

- Antes de dormir.

- Otras.

11) ¿Habló alguna vez con su pediatra de cabecera sobre el uso de estos dispositivos y sus recomendaciones en los niños?

- sí.

- NO. 